\title{
Survival and outcomes following cardiopulmonary resuscitation; a descriptive study in Iran
}

\author{
Ali Arhami Dolatabadi', Elham Memary ${ }^{2 *}$, Majid Shojaee ${ }^{1}$, Hossein Alimohammadi', Hamid Kariman', \\ Ali Shahrami ${ }^{1}$, Abdelrahman Ibrahim Abushouk ${ }^{3}$
}

'Department of Emergency Medicine, Imam Hosein Hospital, Shahid Beheshti University of Medical Sciences, Tehran, Iran ${ }^{2}$ Department of Anesthesiology, Imam Hosein Hospital, Shahid Beheshti University of Medical Sciences, Tehran, Iran ${ }^{3}$ Faculty of Medicine, Ain Shams University, Cairo, Egypt

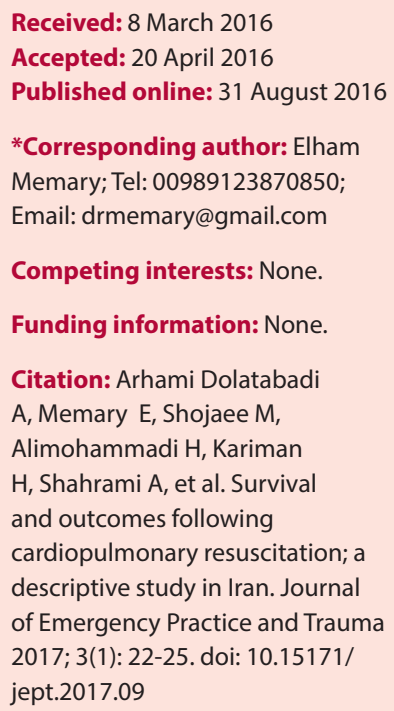

\begin{abstract}
Objective: Cardiopulmonary resuscitation (CPR) has been known in its present form since 1960. Different studies have reported variable outcomes among different countries. Therefore, the purpose of this study was to assess the rate of CPR success and the survival rate in managing cardiac arrest among patients in an educational medical center.

Methods: This cross-sectional study was performed at Imam Hosein hospital, Tehran, Iran. All patients, admitted to the emergency department with cardiac arrest between March 2007 and January 2008 were included. We used a formerly designed registration form and hospital documentation to retrieve the data of included patients. The main outcomes were the rate of CPR success and the survival rate of these patients.

Results: Totally 855 patients were included, from which 510 (59.64\%) were males. The mean age of included patients was $63 \pm 17.6$. The CPR process was successful among $364(42.58 \%)$ patients. A total number of 101 (11.82\%) patients were discharged from the hospital. Different factors as the cause of cardiac arrest and past medical problems affected the probability of CPR success and the survival of patients with cardiac arrest.

Conclusion: Survival rate at hospital discharge was less than one-third of patients and nearly half of the patients received successful CPR. More intensive care unit (ICU) facilities and educational interventions for the emergency staff and the community can enhance the survival of cardiac arrest patients in our health system.

Keywords: Resuscitation, Cardiac arrest, Survival, Outcome, Iran, Emergency department
\end{abstract}

\section{Introduction}

Cardiac arrest is a fatal event that may occur in adults due to different arrhythmias such as pulseless ventricular tachycardia (VT), pulseless electrical activity (PEA), ventricular fibrillation, or cardiac asystole (1-3). Even though the use of closed chest cardiopulmonary resuscitation (CPR), first described in 1960, has widely expanded, survival following a cardiac arrest is usually poor $(4,5)$. Recently, several researches have focused on identifying the factors that impact the patients' probabilities of responding to CPR (6). Some of them compared different survival rates during different timeframes of the day and different days of the week, short-term and long-term prognosis after in-hospital CPR based on patients' age, survival following witnessed and unwitnessed arrest, or type of arrest (7-9). On the other hand, different studies conducted in this field showed that accurate resuscitation rates based on different factors are not the same among different centers. Such data can be used to improve the education level of hospital staff, considering better training program, improvement of care delivery processes, and proper equipment decisions $(8,9)$. Different survival rates at hospital discharge have been reported in different studies conducted in different countries (10-14). The purpose of this study was to assess the rate of CPR success and the survival rate in managing cardiac arrest among patients in an educational medical center.

\section{Methods}

This cross-sectional study was carried out between March 2007 and January 2008 at the emergency department of Imam Hussein hospital, Tehran, Iran. The study population was selected using the census method. All patients who were brought to emergency department due to cardiac arrest or arrested in the hospital and finally received CPR were included. Patients brought to the emergency department who were nearly dead, so they did not receive $\mathrm{CPR}$ and also patients who experienced respiratory arrest without cardiac arrest, were excluded from the study.

A formerly designed data registration form was used to collect the information related to each patient. It included questions about CPR outcome, final status of the patient, duration of CPR, patients' cardiac rhythm before arrest, patients' previous medical problems, and whether or not 
there was a witness arrest. Age, sex, cigarette smoking, drug abuse and alcohol drinking, place and cause of cardiac arrest, were also investigated. Hospital documentation at the emergency ward was used to gather the information needed for each patient. CPR outcome was considered to be successful only if the patient had spontaneous breathing and detectable pulse rate. In order to be sure about their final status, patients were followed to the end of their hospital stay.

All statistical analysis was performed using SPSS software version 11. Descriptive statistical analysis was done for all demographic characteristics, patients' variables and outcomes. Fisher exact test and chi-square test were used to compare differences between groups of patients with successful or unsuccessful CPR and between the patients who survived until hospital discharge or expired during hospital stay. Survival rate was calculated as the number of patients surviving at the time of hospital discharge divided by the total population who received CPR.

\section{Results}

Totally 855 patients were included, from which 510 (59.64\%) cases were males. The mean age was $63 \pm 17.61$. Different variables related to CPR outcome were investigated and the results were as follows:

The CPR process was successful among 364 (42.58\%) patients while it was considered to be unsuccessful among the remaining 491 (57.42\%). A total number of 101 (11.82\%) patients were discharged from the hospital (survival rate at hospital discharge), including both with and without neurologic deficit. Among patients who were survived following CPR until hospital discharge, 81 patients (22.25\%) were discharged without neurologic deficits while 20 (5.5\%) had neurologic deficit at the time of discharge. The remaining 263 (72.25\%) patients, despite having a successful CPR, expired before hospital discharge.

The mean age among patients receiving successful CPR was $60.5 \pm 11.4$ years while this was $65.11 \pm 13.7$ years among those receiving unsuccessful CPR. Considering 63 year as the mean age of patients, $33(6.63 \%)$ of patients discharged from hospital were older than 63 year comparing to $68(18.99 \%)$ of patients younger than 63 year $(P=0.016)$.

A total number of $225(44.11 \%)$ male patients as compared to $139(40.28 \%)$ female patients were considered to have a successful CPR $(P=0.063)$. The number of female patients who were discharged alive was $40(11.59 \%)$ cases while this was $61(11.96 \%)$ cases among male patients $(P=0.10)$. Duration of CPR: The mean time for CPR process was $16.06(\mathrm{SD}=0.999)$ minutes among the patients considered to have a successful CPR and $43.48(\mathrm{SD}=15.75)$ minutes among the others receiving unsuccessful CPR process. Among the patients who were discharged from the hospital, this time was statistically lower compared to those who expired before hospital discharge (mean $=12.8 \mathrm{~min}$ utes, $\mathrm{SD}=6.8, P=0.040$ )

Time of receiving CPR: The number of delivered CPR procedures and its success rate were variable at different hours of the day as illustrated in Figure 1.

Place of cardiac arrest: The site of cardiac arrest occurrence varied among the included patients between emergency department, patients' residents, public places or nursing homes. Further details regarding the frequency of cardiac arrest in different sites and their correlation with the rate of successful CPR procedures to unsuccessful ones are illustrated in Figure 2.

Data analysis showed that the rate of CPR success and survival rate at hospital discharge were statistically higher for in-hospital cardiac arrest compared to arrest occurring in other places ( $P=0.047$ and $P=0.012$, respectively).

Witnessing cardiac arrest: Some cardiac arrest events were witnessed by either the family, emergency staff members or strangers, while others were not. Different CPR outcomes were correlated with the existence and type of the witness as illustrated in Table 1.

Causes of cardiac arrest: The rate of CPR success varied among the patients with different underlying causes of cardiac arrest. The least success rates were reported among the patients whose cardiac arrest followed sepsis or unknown causes. Further details about the frequency of different causes and their relation to CPR success rate are

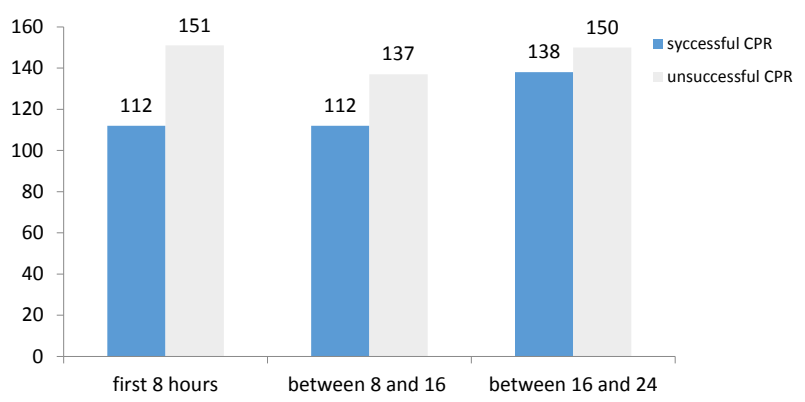

Figure 1. The number of successful and unsuccessful CPR regarding different hours of day.

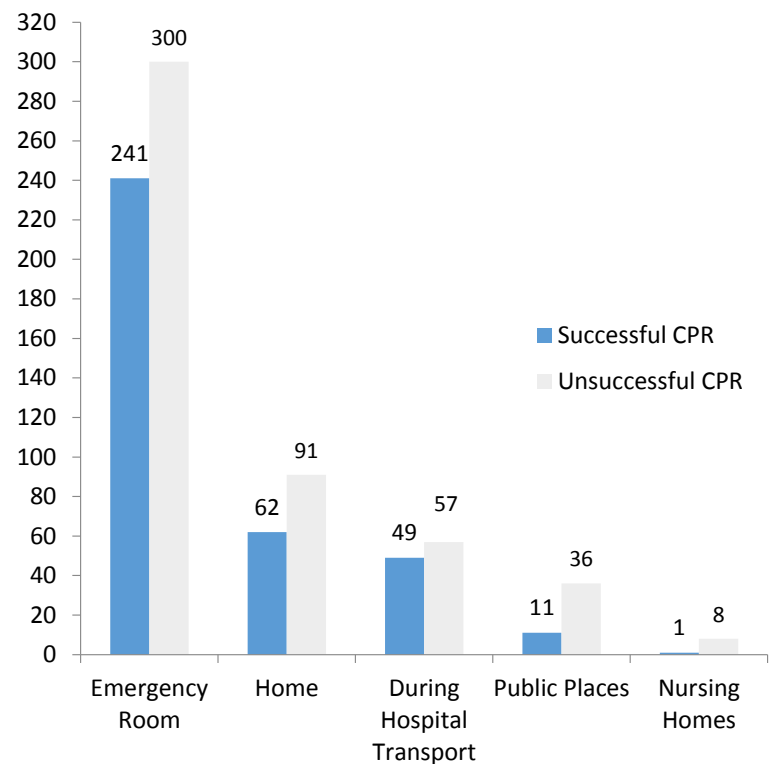

Figure 2. The number of successful and unsuccessful CPR regarding place of cardiac arrest. 
Table 1. Different CPR outcomes regarding witnessed or unwitnessed cardiac arrest

\begin{tabular}{lccc}
\hline & Total frequency (\%) & Successful CPR (\%) & Unsuccessful CPR (\%) \\
\hline Family & $154(18.01)$ & $61(39.61)$ & $93(60.39)$ \\
Staff of emergency ward & $641(74.97)$ & $291(45.39)$ & $350(54.61)$ \\
Strangers & $37(4.32)$ & $6(16.21)$ & $31(83.79)$ \\
No witness & $23(2.69)$ & $5(21.73)$ & $18(28.27)$ \\
\hline
\end{tabular}

Abbreviation: CPR, cardiopulmonary resuscitation.

Table 2. Different Medical problems among patients and different CPR outcome in each one

\begin{tabular}{lccc}
\hline & Total frequency (\%) & Successful CPR (\%) & Unsuccessful CPR (\%) \\
\hline Hypertension & $235(28.17)$ & $93(39.57)$ & $142(60.43)$ \\
History of MI & $67(8.03)$ & $20(29.5)$ & $47(70.5)$ \\
History of IHD & $311(37.29)$ & $141(45.67)$ & $170(45.33)$ \\
\hline Pacemaker & $12(1.43)$ & $6(50)$ & $7(50)$ \\
History of valvular heart disease & $9(1.08)$ & $2(29.22)$ & $96.78)$ \\
History of DM & $137(16.42)$ & $41(29.92)$ & $21(23.86)$ \\
History of CVA & $88(10.5)$ & $67(76.14)$ & $10(28.58)$ \\
Renal disease & $35(4.19)$ & $25(71.42)$ & $33(45.84)$ \\
Asthma and/or COPD & $72(8.63)$ & $39(54.16)$ & $2(80)$ \\
History of Liver disease & $10(1.02)$ & $2(20)$ & $24(77.42)$ \\
Cancer & $31(3.71)$ & $7(22.58)$ & $100 \%$ \\
AIDS & $3(0.35)$ & $0(0.0)$ & \\
\hline
\end{tabular}

Abbreviations: IHD, ischemic heart disease; CVA, cerebral vascular attack; DM, diabetes mellitus; COPD, chronic obstructive pulmonary disease $\mathrm{MI}$, myocardial infarction; CPR, cardiopulmonary resuscitation.

illustrated in Figure 3.

Past medical problems: Included patients had different previous medical problems and the rate of CPR success differed in correlation to them as illustrated in Table 2.

Prior cardiac rhythm: Different cardiac rhythms were detected prior to receiving CPR including asystole (720 patients, $84.21 \%$ ), VF (48 patients, 5.6\%), VT (39 patients, $4.43 \%$ ), PEA (29 patients, $3.47 \%$ ) and bradycardia (18 patients, $2.15 \%)$. Rates of successful CPR was 308 (42.77\%), 31 (67.39\%), 28 (75.63\%), 19 (65.51\%) and 11 (61.11\%) respectively. Chi-square analysis showed no statistically significant correlation between the type of prior cardiac rhythm and CPR success rate or survival rate at hospital discharge ( $P=0.10$ and $P=0.14$, respectively)

Patients' habits in relation to CPR outcome: Our sample included 139 patients $(16.66 \%)$ who were cigarette smokers, among those $49(35.25 \%)$ received successful CPR. Another 71 patients $(8.51 \%)$ were addicted and 28 (39.43\%) of them received successful CPR. Within the included patients, 9 (1.08\%) had drunk alcohol recently, among those $3(33.33 \%)$ received successful CPR.

\section{Discussion}

In our study nearly half of the patients received successful $\mathrm{CPR}$, among those the survival rate at the time of hospital discharge was less than one-third of them.

Furthermore, our study showed that factors including age under 63 years, cardiac arrest occurrence at the emergency department and duration of CPR less than 12.8 minutes were associated with a higher rate of survival at hospital discharge. The only factor which was associated with a higher rate of successful CPR was cardiac arrest occurring at the emergency department. Neither the sex, nor the car-

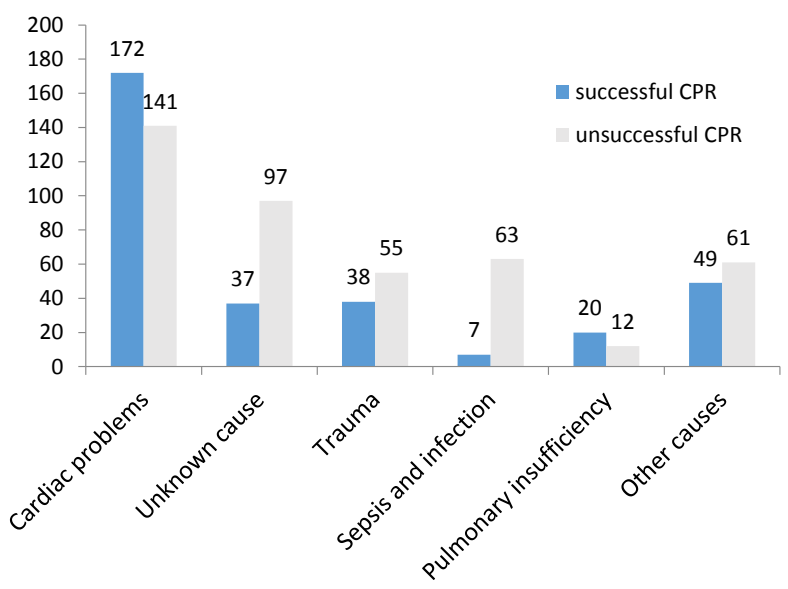

Figure 3. The number of successful and unsuccessful CPR considering different causes of cardiac arrest.

diac rhythm prior to CPR had any effect on the outcome of CPR or survival rate at hospital discharge.

When compared to other international studies, a study conducted in the United States from 1992 to 2005, findings indicated that the survival rate at discharge was $18.3 \%$ in US hospitals during that time period (13). Similarly, in a descriptive study conducted between 2003 and 2006 in Spain, survival at hospital discharge was reported to be $20 \%$ (10). Another retrospective study done on 383 patients between 1998 and 2003 in Pakistan, showed that survival at hospital discharge was 19\% (11). Moreover, another study conducted in a developing country between 2003 and 2006 showed that only $7.4 \%$ of patients survived at discharge (14). Compared to this study, a former prospective study conducted on 206 patients during the year of 2002 in Kashan, Iran, reported a survival rate of 5.3\% at 
hospital discharge (12). In comparison to all of these findings, our study seems to have an acceptable survival rate among developing countries. Despite being lower than those in developed countries, it seems that the survival rate has increased in Iran over the past 6 years.

\section{Implications for practice}

Despite the successful CPR procedure in nearly half of the patients, only $11.82 \%$ of them survived at hospital discharge. Considering that most patients who receive a successful CPR procedure need to be admitted to an ICU, this finding can be attributed to inadequate ICU care in our hospital or insufficient involvement of different health services. In other words, it can be concluded that the resuscitation process in our country needs to be more specialized and further attempts should be conducted to improve it. This improvement should focus both on developing hospital facilities and augmenting the educational process related to emergency conditions. Facilities like more ICU beds and developing specialized rooms for shock management in hospitals can be highly effective in managing patients after CPR. Moreover, since different factors including the knowledge about emergency conditions can influence the survival rate, developing educational programs, not only for hospital staffs but also for the community would lead to better management of these conditions.

\section{Strengths and Limitations}

Our study was conducted in one of the largest university hospitals in Tehran. This can serve as a strength point or a limitation to our findings. The emergency ward in this hospital is supported by specialists of emergency medicine, so the findings may differ from those hospitals with no specialists in this field. On the other hand, this hospital is one of the referral hospitals for emergency conditions, so different patients with different characteristics were included in our study. Although the large number of patients included in this study would serve as a strength point, involving just one hospital for sampling can be considered as a limitation to our study.

\section{Conclusion}

Survival rate at hospital discharge was less than one-third of patients and nearly half of the patients received successful CPR. More ICU facilities and educational interventions for the emergency staff and the community can enhance the survival of cardiac arrest patients in our health system.

\section{Ethical issues}

The protocol of study was approved by ethical committee of Shahid Beheshti University of Medical Sciences. The researchers were adhered to Helsinki - Ethical Principles for medical research involving human subjects..

\section{Authors' contributions}

All authors met the four criteria for authorship based on the recommendations of the International Committee of Medical Journal Editors.

\section{References}

1. Nadkarni VM, Larkin GL, Peberdy MA, Carey SM, Kaye W, Mancini ME, et al. First documented rhythm and clinical outcome from in-hospital cardiac arrest among children and adults. JAMA 2006; 295(1): 50-57. doi: 10.1001/ jama.295.1.50.

2. Kariman H, Harati S, Safari S, Baratloo A, Pishgahi M. Validation of EGSYS score in prediction of cardiogenic syncope. Emerg Med Int. 2015; 2015: 515370. doi: 10.1155/2015/515370.

3. Arhami Dolatabadi A, Kashani P, Hatamabadi H, Kariman $\mathrm{H}$, Baratloo A. Using risk factors to help in the diagnosis of acute myocardial infarction in patients with non-diagnostic electrocardiogram changes in emergency department. Journal of Emergency Practice and Trauma 2014; 1(1): 3-6.

4. Kouwenhoven WB, Jude JR, Knickerbocker GG. Closedchest cardiac massage. JAMA 1960; 173: 1064-67.

5. Ebell MH, Becker LA, Barry HC, Hagen M. Survival after in-hospital cardiopulmonary resuscitation. A metaanalysis. J Gen Intern Med 1998; 13(12): 805-16.

6. Rosenberg M, Wang C, Hoffman-Wilde S, Hickam D. Results of cardiopulmonary resuscitation. Failure to predict survival in two community hospitals. Arch Intern Med 1993; 153(11): 1370-5.

7. Di Bari M, Chiarlone M, Fumagalli S, Boncinelli L, Tarantini F, Ungar A, et al. Cardiopulmonary resuscitation of older, inhospital patients: immediate efficacy and longterm outcome. Crit Care Med 2000; 28(7): 2320-5.

8. Peberdy MA, Ornato JP, Larkin GL, Braithwaite RS, Kashner TM, Carey SM, et al. Survival from in-hospital cardiac arrest during nights and weekends. JAMA 2008; 299(7): 785-92. doi: 10.1001/jama.299.7.785.

9. Brindley PG, Markland DM, Mayers I, Kutsogiannis DJ. Predictors of survival following in-hospital adult cardiopulmonary resuscitation. CMAJ 2002; 167(4): 343-8.

10. Ezquerra García AM, Suberviola Fernández I, Pavía Pesquera MC. Evaluation of the effectiveness of an inhospital cardiac alarm system. Enferm Intensiva 2009; 20(2): 58-68. [In Spanish].

11. Khan NU, Razzak JA, Ahmed H, Furqan M, Saleem AF, Alam $\mathrm{H}$, et al. Cardiopulmonary resuscitation: outcome and its predictors among hospitalized adult patients in Pakistan. Int J Emerg Med 2008; 1(1): 27-34. doi: 10.1007/ s12245-008-0016-4.

12. Hajbaghery MA, Mousavi G, Akbari H. Factors influencing survival after in-hospital cardiopulmonary resuscitation. Resuscitation 2005; 66(3): 317-21. doi: 10.1016/j. resuscitation.2005.04.004.

13. Ehlenbach WJ, Barnato AE, Curtis JR, Kreuter W, Koepsell TD, Deyo RA, et al. Epidemiologic study of in-hospital cardiopulmonary resuscitation in the elderly. $\mathrm{N}$ Engl J Med 2009; 361(1): 22-31. doi: 10.1056/NEJMoa0810245.

14. Krittayaphong R, Saengsung $P$, Chawaruechai $T$, Yindeengam A, Udompunturak S. Factors predicting outcome of cardiopulmonary resuscitation in a developing country: the Siriraj cardiopulmonary resuscitation registry. J Med Assoc Thai 2009; 92(5): 618-23. 